\section{Part 2. Adult basic life support: 2015 Korean Guidelines for Cardiopulmonary Resuscitation}

\author{
Keun Jeong Song ${ }^{1}$, Jae-Bum Kim², Jinhee $\mathrm{Kim}^{3}$, Chanwoong Kim${ }^{4}$, \\ Sun Young Park ${ }^{5}$, Chang Hee Lee ${ }^{6}$, Yong Soo Jang $^{7}$, Gyu Chong Cho ${ }^{8}$, \\ Youngsuk $\mathrm{Cho}^{8}$, Sung Phil Chung ${ }^{9}$, Sung Oh Hwang ${ }^{10}$

\begin{abstract}
'Department of Emergency Medicine, Samsung Medical Center, Sungkyunkwan University School of Medicine, Seoul, Korea

${ }^{2}$ Department of Thoracic and Cardiovascular Surgery, Keimyung University School of Medicine, Daegu, Korea

${ }^{3}$ Department of Anesthesiology, Seoul National University Bundang Hospital, Seongnam, Korea ${ }^{4}$ Department of Emergency Medicine, Chung-Ang University College of Medicine, Seoul, Korea ${ }^{5}$ Department of Nursing Science, Baekseok University, Cheonan, Korea

${ }^{6}$ Department of Emergency Medical Service, Namseoul University, Cheonan, Korea

${ }^{7}$ Department of Emergency Medicine, Hallym University Kangnam Sacred Heart Hospital, Seoul, Korea

${ }^{8}$ Department of Emergency Medicine, Hallym University Kangdong Sacred Heart Hospital, Seoul, Korea

${ }^{9}$ Department of Emergency Medicine, Yonsei University College of Medicine, Seoul, Korea

${ }^{10}$ Department of Emergency Medicine, Yonsei University Wonju College of Medicine, Wonju, Korea
\end{abstract}

elSSN: 2383-4625

Received: 16 February 2016

Revised: 19 March 2016

Accepted: 19 March 2016

Correspondence to: Keun Jeong Song Department of Emergency Medicine, Samsung Medical Center, Sungkyunkwan University School of Medicine, 81 Irwon-ro Gangnam-gu, Seoul 06351, Korea

E-mail: emsong@skku.edu

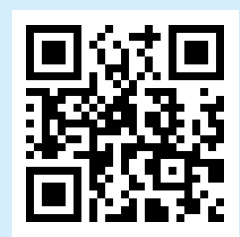
of cardiac arrest can be prevented by reducing the risk factors of cardiac and cerebrovascular diseases in adults. ${ }^{1}$

Efforts need to be made to reduce these risk factors and precipitating factors that cause cardiac arrest. Inside of a hospital, the signs and symptoms presenting prior to cardiac arrest need to be rapidly recognized and treated. The medical emergency team or rapid response team have a role in this process. ${ }^{2,3}$ Lay person should be taught about signs or symptoms of cardiac arrest, which will increase their ability to recognize cardiac arrest.

\section{Early access}

The second link in the chain of survival is early access which includes making a phone call to the 119 EMS center and activating EMS. A bystander recognizing cardiac arrest calls the 119 emer-
Chain of survival is a series of steps required to increase the survival rate of patients with cardiac arrest. To increase the survival rate of patients with cardiac arrest, chain of survival should be strengthened in a community. Chain of survival in 2015 Korean cardiopulmonary resuscitation (CPR) guidelines is composed of five chains: the prevention and immediate recognition of cardiac arrest, early access (activation of emergency medical system [EMS]), early CPR, early defibrillation, and effective advanced life support and post-cardiac arrest care (Fig. 1).

1. The prevention and immediate recognition of cardiac arrest

The new concept of preventing and immediately recognizing cardiac arrest was newly introduced in 2015 Korean CPR guidelines as the first link in the chain of survival. The development
How to cite this article: Song KJ, Kim JB, Kim J, Kim C, Park SY, Lee $\mathrm{CH}$, Jang YS, Cho GC, Cho Y, Chung SP, Hwang SO. Part 2. Adult basic life support: 2015 Korean Guidelines for Cardiopulmonary Resuscitation. Clin Exp Emerg Med 2016;3(S):S10-S16.

This is an Open Access article distributed under the terms of the Creative Commons Attribution Non-Commercial License (http:// creativecommons.org/licenses/by-nc/3.0/). 
gency medical services and reports an incidence of cardiac arrest. The emergency medical dispatcher who receives the call dispatches an ambulance and emergency personnel to the scene. The emergency medical dispatcher should have an ability to instruct CPR via telephone to the bystander.

\section{Early CPR}

Immediately after the call to 119 EMS center, a bystander should immediately start and continue CPR until emergency personnel arrive. CPR performed by a bystander increases the survival rate of the victims with cardiac arrest. ${ }^{4}$ To increase the rate of bystander CPR, schools, militaries, residential areas, work places, and public institutions need to provide education on CPR.

\section{Early defibrillation}

Defibrillation is the most important intervention to treat ventricular fibrillation. Defibrillation is more effective when it is performed early after ventricular fibrillation develops. ${ }^{5}$ An automated external defibrillator (AED) is used for early defibrillation. If an AED is installed in public places through a public access defibrillation program, the survival rate of patients with ventricular fibrillation remarkably increases with early defibrillation. ${ }^{6}$ AED can be safely used by lay persons after they receives a brief instruction to use.

\section{Effective advanced life support and post-cardiac arrest care}

Effective advanced life support needs to be performed to restore the spontaneous circulation of patients with cardiac arrest. Integrated post-cardiac arrest care is required for patients whose spontaneous circulation has recovered from cardiac arrest. Post-cardiac arrest care is an integrated treatment process that includes general intensive care, targeted temperature management, percutaneous coronary intervention for acute coronary syndrome, and the seizure control. ${ }^{7-9}$ Patients resuscitated from cardiac ar- rest need to be transported to a medical center or facility where integrated post-cardiac arrest care including targeted temperature management or percutaneous coronary intervention can be performed. ${ }^{10}$

\section{MAJOR UPDATE OF THE 2015 KOREAN BASIC LIFE SUPPORT GUIDELINES}

The main contents newly added or revised in the 2015 Korean basic life support (BLS) guideline are as follows: (1) Cardiac arrest should be immediately evaluated by the presence of apnea or abnormal respiration. Abnormal respiration represents all forms of respiration that are not normal, such as apnea or agonal gasps." (2) Healthcare providers also need to simultaneously check the patient's pulse and respiration within 10 seconds, and they should not delay compression to check for the absence or presence of a pulse. (3) Regarding the order of CPR, chest compression needs to be performed prior to breathing. Similar to the guideline in 2011, the order of compression-airway-breathing (C-A-B sequence) needs to be maintained. ${ }^{11,12}$ (4) High quality CPR is important. It is suggested that in adult patients with cardiac arrest, the depth of compression should be approximately $5 \mathrm{~cm}$, and the rate should be $100-120 / \mathrm{min}$. It is recommended that the interruption of compression is minimized to less than 10 seconds, and breathing should not be excessively performed. ${ }^{11-18}$ (5) Healthcare providers, including 119 emergency medical technicians, should always perform CPR with both compression and ventilation.

\section{ADULT BLS FOR LAY RESCUERS}

2015 Korean CPR guidelines recommend compression-only CPR when a non-healthcare professional lay person rescues a victim with cardiac arrest. The steps of BLS consist of determining unresponsiveness, calling the 119 emergency dispatcher center, per-

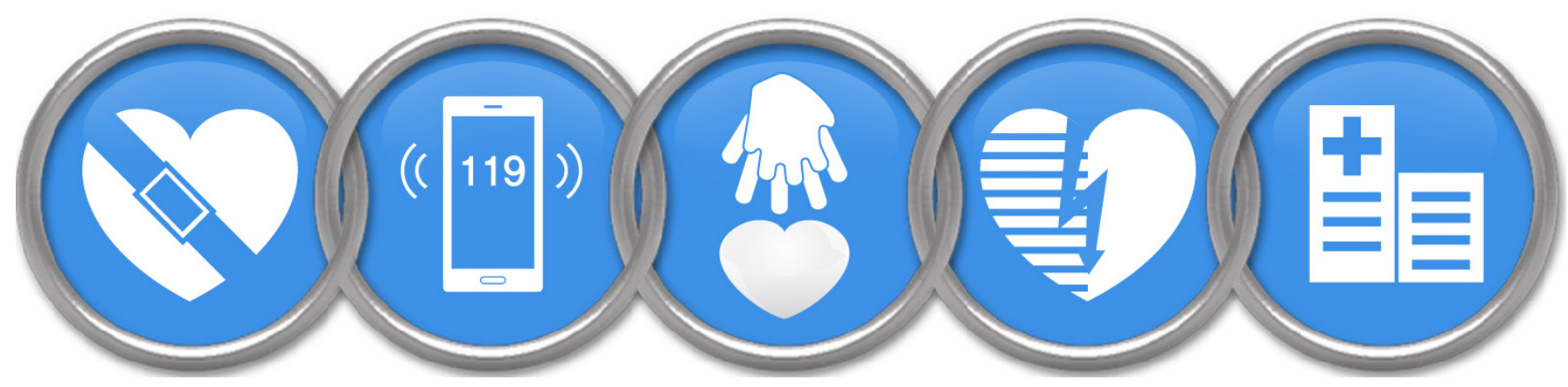

Fig. 1. Chain of survival in 2015 Korean Guidelines for Cardiopulmonary Resuscitation. Components of chain of survival are the prevention and immediate recognition of cardiac arrest, early access, early cardiopulmonary resuscitation, early defibrillation, and effective advanced life support and post-cardiac arrest care. 


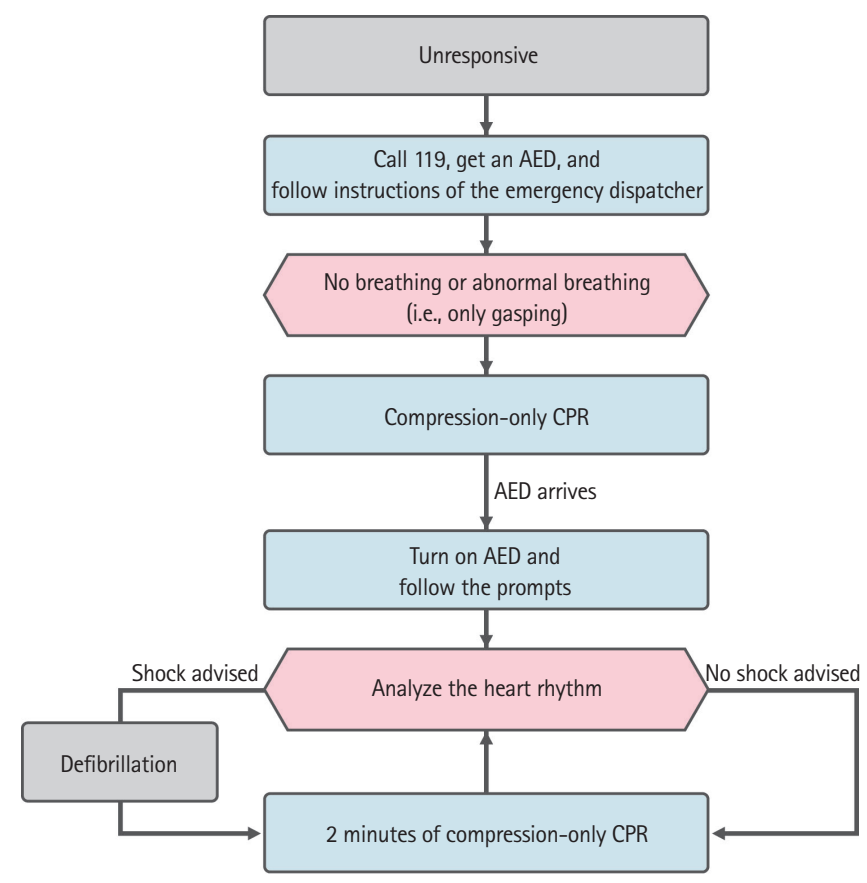

Fig. 2. Basic life support algorithm for lay persons. AED, automated external defibrillator; $C P R$, cardiopulmonary resuscitation.

forming compression-only CPR, and using an AED. The sequence of adult BLS for lay persons is summarized in BLS algorithm for lay rescuers (Fig. 2).

\section{Check for responsiveness}

The first step of BLS is to check for responsiveness. The safety of the scene needs to be assessed first before approaching the collapsed person. Responsiveness can be evaluated by shouting, "Are you alright?" to the collapsed person, and by tapping his/her shoulder.

\section{119 Emergency call}

If the collapsed person is unresponsive, a 119 emergency call should be made immediately, and an AED needs to be requested. If one witnesses a collapsed person, he/she needs to request that someone call 119 by loudly asking others nearby for help. One should also call 119 if there is no one else around. When calling 119, the location of the incidence, situation of the incidence, the patient's status, and emergency treatment that is being conducting needs to be reported. ${ }^{1}$

\section{Assessing respiration (recognition of cardiac arrest)}

The patient's respiration should be evaluated after checking responsiveness and making the 119 call. Patients who are unresponsive and have apnea or abnormal respiration (e.g., agonal respiration) should be determined to be in cardiac arrest. It is challenging for a lay rescuer to precisely evaluate the patient's respiration status. ${ }^{19}$ Thus, a lay rescuer needs the help of an emergency medical dispatcher to determine the respiration status and to provide guidance on how to perform compression-only CPR. ${ }^{20}$

\section{Compression-only CPR}

It is recommended that lay rescuers perform compression-only CPR for adult victims with cardiac arrest. The blood oxygen concentration does not rapidly decrease when cardiac arrest suddenly occurs without hypoxia such as in cases of cardiac arrest from cardiac origin.; instead, it may maintain for a few minutes at the early stage of cardiac arrest. ${ }^{21}$ Therefore, ventilation may not be necessary during the early stage of cardiac arrest. According to a series of recent clinical trials, the survival rate increases with compression-only CPR compared to no CPR. ${ }^{22,23}$ Also, compressiononly CPR has a similar survival rate compared to standard CPR that includes ventilation in patients with cardiac arrest of nonrespiratory origin. ${ }^{24}$ Standard CPR is recommended if the rescuer knows how to ventilate and is willing to provide ventilation.

In adult cardiac arrest, the depth of compression should be approximately $5 \mathrm{~cm}$, and the rate should be maintained at 100-120/ min. ${ }^{15,25}$ The recommended position of one's hand when performing compression is at the lower half of the sternum. If the depth of compression is greater than $6 \mathrm{~cm}$, there is a possibility of increasing incidence of complications. ${ }^{26}$

\section{AED use}

An AED should be used immediately when it arrives. If an AED arrives while CPR is being performed, one needs to push the power button to turn it on. After taking off the patient's shirt, two pads need to be firmly attached to the patient's bare chest. Chest compressions need to be stopped while the AED analyzes the cardiac rhythm of the patient with cardiac arrest. If defibrillation is required, the AED says, "need defibrillation" and charges the defibrillator by itself. If a voice or screen instruction says, "push the shock button" after charging, one must ensure that no one touches the patient for safety purposes, and then the shock button can be pushed. One must resume chest compressions immediately after defibrillation.

\section{DISPATCHER-ASSISTED CPR}

The emergency medical dispatcher can assist a lay rescuer to recognize cardiac arrest and instruct CPR. An emergency medical dispatcher asks the caller if the collapsed person is responsive and if his/her respiration is abnormal. ${ }^{27}$ An emergency medical dispatcher determines cardiac arrest in cases in which the collapsed person is unresponsive and has apnea or abnormal respi- 


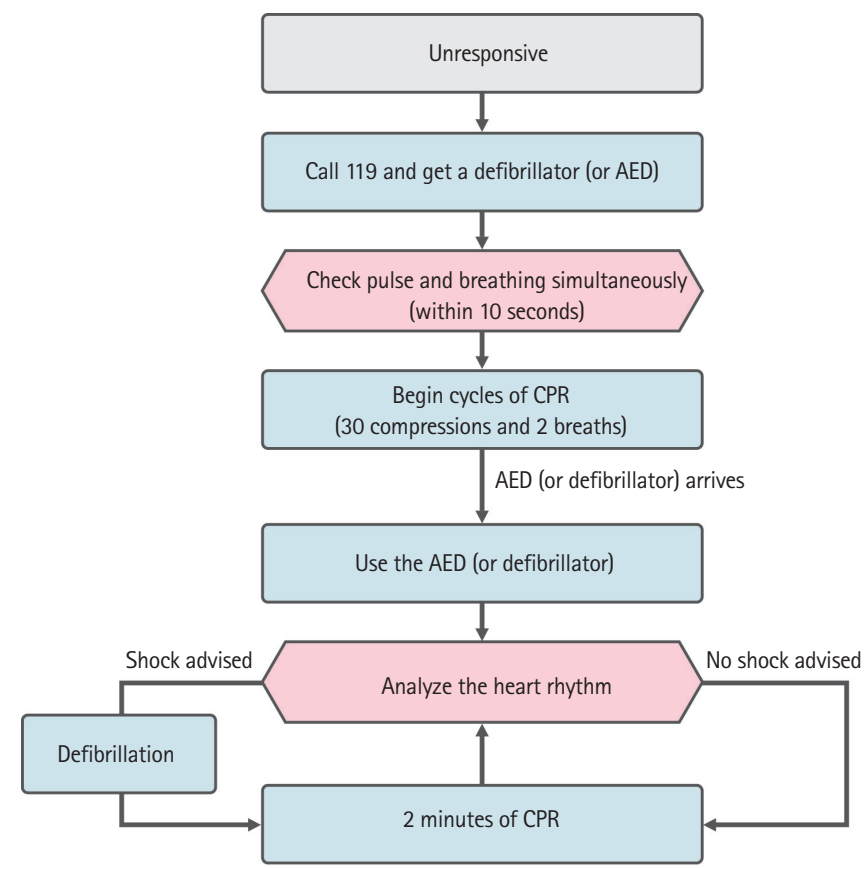

Fig. 3. Basic life support algorithm for healthcare providers. AED, automated external defibrillator; CPR, cardiopulmonary resuscitation.

ration. If an emergency medical dispatcher recognizes cardiac arrest, the dispatcher will provide instructions on how to perform CPR via telephone. Instruction of compression-only CPR to lay rescuers by emergency dispatchers increases bystander CPR rate and improves the survival rate of victims with cardiac arrest. ${ }^{27-29}$ If the patient with cardiac arrest is submerged or hypoxic, or the rescuer is able to perform breathing, both chest compression and breathing need to be performed.

\section{EXEMPTION OF THE LIABILITY IN THE KOREAN EMERGENCY MEDICAL SERVICE LAW}

There is an exemption clause in the Korean Emergency Medical Service Law about liability of the rescuer who provides emergency treatment. The fifth subsection and second article of this law articulates the following: "When a person renders first aid or emergency care to an emergency patient whose life is at stake and there is no intentional or severe fault on property damage or injuries as a result of his/her acts, he/she shall not be liable for any civil damages and has no criminal liability including for the victim's death". ${ }^{30}$ This law protects good Samaritans.

\section{BLS FOR HEALTHCARE PROVIDERS}

The steps of BLS for healthcare providers consist of determining unresponsiveness, calling the 119 emergency dispatcher center, checking for breathing and pulse, performing CPR, and using an AED or defibrillator. The sequence of adult BLS for healthcare providers is summarized in BLS algorithm for healthcare providers (Fig. 3).

1. Check unresponsiveness, call the 119 dispatcher center As in BLS for lay persons, the first step of BLS is to check for unresponsiveness. The safety of the scene needs to be assessed first before approaching the collapsed person. Unresponsiveness can be evaluated by shouting, "Are you alright?" to the collapsed person, and by tapping his/her shoulder.

\section{Assessing respiration and pulse}

The patient's respiration should be evaluated after determining responsiveness and making the 119 call. Determination of the absence or presence of a pulse and respiration should be performed within 10 seconds. To determine the pulse of an adult patient with cardiac arrest, the carotid artery needs to be assessed. ${ }^{11}$

\section{Chest compression}

In adult cardiac arrest, the depth of compression should be approximately $5 \mathrm{~cm}$, and the rate should be maintained at 100-120/ min. $^{15,25}$ The recommended position of one's hand when performing compression is at the lower half of the sternum. If the depth of compression is greater than $6 \mathrm{~cm}$, there is a possibility of increasing complications. ${ }^{26}$ The recoil of the chest needs to be maximized after each compression. ${ }^{31,32}$ The suggested compressionventilation ratio is $30: 2 .{ }^{33-35}$ Considering the high quality of CPR and the fatigue of a rescuer, a role of compressor needs to rotate every 2 minutes. ${ }^{11}$ The interruption of compression should be minimized to less than 10 seconds during CPR. ${ }^{16,18,36}$

In the case that an advanced airway is in place, it is suggested that one rescuer continues compression at a rate of 100-120/min without interruption, and the other rescuer provides respiration with a bag valve mask every 6 seconds (10 breaths/min). Healthcare providers, including 119 emergency medical technicians, should always perform CPR with both compression and ventilation. ${ }^{11,21,25}$

\section{Airway and rescue breathing}

When healthcare providers open the airway of patients with cardiac arrest and no evidence of injuries to the head or neck, the airway should always be maintained by using the head tilt-chin lift maneuver. If a cervical injury is suspected, the jaw thrust technique, which does not include head extension, should be used to open the airway.

Mouth-to-mouth ventilation is recommended to provide res- 
cue breathing to the patient. We recommend to ventilate the patient with inspiration duration of 1 second and with the tidal volume as the patient's rising chest is visually identified. Provide ventilation once every 6 seconds ( 10 breaths $/ \mathrm{min}$ ) if there are two or more healthcare providers and an advanced airway is inserted. Avoid hyperventilation during rescue breathing.

The tidal volume should be maintained at 500 to $600 \mathrm{~mL}$ (6-7 $\mathrm{mL} / \mathrm{kg}$ ) during adult CPR. ${ }^{37-39}$ The most common cause of failure in ventilation is inappropriate opening of the airway. If the patient's chest does not rise during the first attempt of breathing, breathing should be conducted again after properly performing the head tilt-chin lift maneuver. When respiration assistance is needed for a patient with spontaneous circulation (e.g., when a strong pulse is palpable), ventilation should be performed every 5 to 6 seconds or $10-12 / \mathrm{min}$. Bag mask ventilation is a method to provide positive pressure ventilation without an advanced airway. A rescuer provides about 500 to $600 \mathrm{~mL}$ of tidal volume using an adult bag mask. This should be performed for 1 second, and oxygen (concentration greater than 40\%, minimum 10-12 L/min) should be provided if possible. ${ }^{40} \mathrm{It}$ is most effective when two or more experienced rescuers use the bag mask.

After the advanced airway is placed, chest compression should be performed at 100-120/min without interruption, and ventilation should be performed every 6 seconds ( 10 breaths/min). Healthcare providers can choose either a bag mask or advanced airway while CPR is performed in adult patients with cardiac arrest. To provide an advanced airway, either a supraglottic airway or endotracheal tube can be placed.

\section{AED use}

An AED should be used immediately when it arrives. If an AED arrives while CPR is being performed, one rescuers use an AED and the other continues CPR. Chest compression should not be stopped except a period for analyzing rhythm and delivering a shock. After delivering a shock, immediately restart chest compression. After every 2 minutes of CPR, analyze the cardiac rhythm and check the patient's status. Advanced life support should be started if available.

\section{ACKNOWLEDGMENTS}

Collaborators have contributed to the review of the scientific evidence. The collaborators' are as follows: Chang Je Park, SMG-SNU Boramae Medical Center, Seoul, Korea; Dae Jong Choi, Korean Red Cross, Seoul, Korea; Dong Won Kim, Hallym University, Chuncheon, Korea; Hee Jeong Kim, Baekseok University, Cheonan, Korea; Hyun Jung Kim, Daewon University College, Jecheon, Korea;
Jin Hyuck Lee, Hallym University, Seoul, Korea; Jin Uk Kim, Kyungdong University, Wonju, Korea; Jin Woo Kim, Daejeon Health Sciences College, Daejeon, Korea; Kang Nim Kim, Hallym University Kangdong Sacred Heart Hospital, Seoul, Korea; Myung Lyeol Lee, Daewon University College, Jecheon, Korea; Nam Sik Yoon, Chonnam National University, Gwangju, Korea; Sang Wook Park, Chonnam National University Hospital, Gwangju, Korea; So Hyeon Song, Daegu Health College, Daegu, Korea; Su Youn Kim, Gangdong University, Icheon, Korea.

\section{REFERENCES}

1. Goyal V, Jassal DS, Dhalla NS. Pathophysiology and prevention of sudden cardiac death. Can J Physiol Pharmacol 2016;94: 237-44.

2. Salvatierra G, Bindler RC, Corbett C, Roll J, Daratha KB. Rapid response team implementation and in-hospital mortality. Crit Care Med 2014;42:2001-6.

3. Aneman A, Frost SA, Parr MJ, Hillman KM. Characteristics and outcomes of patients admitted to ICU following activation of the medical emergency team: impact of introducing a twotier response system. Crit Care Med 2015;43:765-73.

4. Hansen $\mathrm{CM}$, Kragholm K, Granger CB, et al. The role of bystanders, first responders, and emergency medical service providers in timely defibrillation and related outcomes after out-of-hospital cardiac arrest: results from a statewide registry. Resuscitation 2015;96:303-9.

5. Holmberg $M$, Holmberg $S$, Herlitz J. Incidence, duration and survival of ventricular fibrillation in out-of-hospital cardiac arrest patients in sweden. Resuscitation 2000;44:7-17.

6. Strohle M, Paal P, Strapazzon G, Avancini G, Procter E, Brugger H. Defibrillation in rural areas. Am J Emerg Med 2014;32: 1408-12.

7. Nielsen N, Wise MP, Cronberg T. Targeted temperature management for cardiac arrest: reply. JAMA Neurol 2015;72:1076-7.

8. Rab T, Kern KB, Tamis-Holland JE, et al. Cardiac arrest: a treatment algorithm for emergent invasive cardiac procedures in the resuscitated comatose patient. J Am Coll Cardiol 2015; 66:62-73.

9. Rittenberger JC, Doshi AA, Reynolds JC; Post Cardiac Arrest Service. Postcardiac arrest management. Emerg Med Clin North Am 2015;33:691-712.

10. Roberts BW, Kilgannon JH, Mitchell JA, et al. Emergency department inter-hospital transfer for post-cardiac arrest care: initial experience with implementation of a regional cardiac resuscitation center in the United States. Resuscitation 2013; 84:596-601. 
11. Berg RA, Hemphill R, Abella BS, et al. Part 5. Adult basic life support: 2010 American Heart Association Guidelines for Cardiopulmonary Resuscitation and Emergency Cardiovascular Care. Circulation 2010;122(18 Suppl 3):S685-705.

12. Marsch S, Tschan F, Semmer NK, Zobrist R, Hunziker PR, Hunziker $\mathrm{S}$. $A B C$ versus $C A B$ for cardiopulmonary resuscitation: a prospective, randomized simulator-based trial. Swiss Med Wkly 2013;143:w13856.

13. Kern KB, Hilwig RW, Berg RA, Sanders AB, Ewy GA. Importance of continuous chest compressions during cardiopulmonary resuscitation: improved outcome during a simulated single lay-rescuer scenario. Circulation 2002;105:645-9.

14. Vadeboncoeur T, Stolz U, Panchal $A$, et al. Chest compression depth and survival in out-of-hospital cardiac arrest. Resuscitation 2014;85:182-8.

15. Stiell IG, Brown SP, Nichol G, et al. What is the optimal chest compression depth during out-of-hospital cardiac arrest resuscitation of adult patients? Circulation 2014;130:1962-70.

16. Beesems SG, Wijmans L, Tijssen JG, Koster RW. Duration of ventilations during cardiopulmonary resuscitation by lay rescuers and first responders: relationship between delivering chest compressions and outcomes. Circulation 2013;127:1585-90.

17. Jost $D$, Degrange $H$, Verret $C$, et al. DEFI 2005: a randomized controlled trial of the effect of automated external defibrillator cardiopulmonary resuscitation protocol on outcome from out-of-hospital cardiac arrest. Circulation 2010;121:1614-22.

18. Cheskes S, Schmicker RH, Christenson J, et al. Perishock pause: an independent predictor of survival from out-of-hospital shockable cardiac arrest. Circulation 2011;124:58-66.

19. Perkins GD, Walker G, Christensen K, Hulme J, Monsieurs KG. Teaching recognition of agonal breathing improves accuracy of diagnosing cardiac arrest. Resuscitation 2006;70:432-7.

20. Tanaka Y, Taniguchi J, Wato Y, Yoshida Y, Inaba H. The continuous quality improvement project for telephone-assisted instruction of cardiopulmonary resuscitation increased the incidence of bystander CPR and improved the outcomes of outof-hospital cardiac arrests. Resuscitation 2012;83:1235-41.

21. Sayre MR, Berg RA, Cave DM, et al. Hands-only (compressiononly) cardiopulmonary resuscitation: a call to action for bystander response to adults who experience out-of-hospital sudden cardiac arrest: a science advisory for the public from the American Heart Association Emergency Cardiovascular Care Committee. Circulation 2008;117:2162-7.

22. SOS-KANTO study group. Cardiopulmonary resuscitation by bystanders with chest compression only (SOS-KANTO): an observational study. Lancet 2007;369:920-6.

23. Abe T, Tokuda Y, Ishimatsu S; SOS-KANTO study group. Pre- dictors for good cerebral performance among adult survivors of out-of-hospital cardiac arrest. Resuscitation 2009;80:431-6.

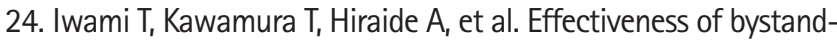
er-initiated cardiac-only resuscitation for patients with outof-hospital cardiac arrest. Circulation 2007;116:2900-7.

25. Stiell IG, Brown SP, Christenson J, et al. What is the role of chest compression depth during out-of-hospital cardiac arrest resuscitation? Crit Care Med 2012;40:1192-8.

26. Moller Nielsen A, Rasmussen LS. Damage and depth of chest compressions. Resuscitation 2013;84:713-4.

27. Becker LB, Pepe PE. Ensuring the effectiveness of communitywide emergency cardiac care. Ann Emerg Med 1993;22(2 Pt 2):354-65.

28. Svensson L, Bohm K, Castren M, et al. Compression-only CPR or standard CPR in out-of-hospital cardiac arrest. N Engl J Med 2010;363:434-42.

29. Iwami T, Kitamura T, Kiyohara K, Kawamura T. Dissemination of chest compression-only cardiopulmonary resuscitation and survival after out-of-hospital cardiac arrest. Circulation 2015; 132:415-22.

30. El Hajj SC, Bordelon CJ, Glancy DL. ECG case of the month: out-of-hospital cardiac arrest. DIAGNOSIS: a trial fibrillation with a rapid ventricular response (150 beats/minute) and right bundle branch block. J La State Med Soc 2014;166:176-8.

31. Zuercher M, Hilwig RW, Ranger-Moore J, et al. Leaning during chest compressions impairs cardiac output and left ventricular myocardial blood flow in piglet cardiac arrest. Crit Care Med 2010;38:1141-6.

32. Yannopoulos D, McKnite S, Aufderheide TP, et al. Effects of incomplete chest wall decompression during cardiopulmonary resuscitation on coronary and cerebral perfusion pressures in a porcine model of cardiac arrest. Resuscitation 2005;64:36372.

33. Gazmuri RJ, Ayoub IM, Radhakrishnan J, Motl J, Upadhyaya MP. Clinically plausible hyperventilation does not exert adverse hemodynamic effects during CPR but markedly reduces end-tidal PCO2. Resuscitation 2012;83:259-64.

34. Kill C, Galbas M, Neuhaus $C$, et al. Chest compression synchronized ventilation versus intermitted positive pressure ventilation during cardiopulmonary resuscitation in a pig model. PLoS One 2015;10:e0127759.

35. Abella BS, Alvarado JP, Myklebust $H$, et al. Quality of cardiopulmonary resuscitation during in-hospital cardiac arrest. JAMA 2005;293:305-10.

36. Cheskes S, Schmicker RH, Verbeek PR, et al. The impact of perishock pause on survival from out-of-hospital shockable cardiac arrest during the Resuscitation Outcomes Consortium 
PRIMED trial. Resuscitation 2014;85:336-42.

37. Wenzel V, Keller C, Idris AH, Dorges V, Lindner KH, Brimacombe $J R$. Effects of smaller tidal volumes during basic life support ventilation in patients with respiratory arrest: good ventilation, less risk? Resuscitation 1999;43:25-9.

38. Dorges V, Ocker $H$, Hagelberg $S$, Wenzel V, Idris AH, Schmucker P. Smaller tidal volumes with room-air are not sufficient to ensure adequate oxygenation during bag-valve-mask ventila- tion. Resuscitation 2000;44:37-41.

39. Dorges V, Ocker H, Hagelberg S, Wenzel V, Schmucker P. Optimisation of tidal volumes given with self-inflatable bags without additional oxygen. Resuscitation 2000;43:195-9.

40. Kilgannon JH, Jones $A E$, Shapiro $\mathrm{NI}$, et al. Association between arterial hyperoxia following resuscitation from cardiac arrest and in-hospital mortality. JAMA 2010;303:2165-71. 